\title{
Special issue on Genome Biology jointly published by Genome and Biochemistry and Cell Biology
}

\author{
Lead Guest Editors \\ James R. Davie, University of Manitoba, Winnipeg, MB, Canada \\ Michael J. Hendzel, University of Alberta, Edmonton, AB, Canada \\ Guest Editors \\ Tasnim H. Beacon, University of Manitoba, Winnipeg, MB, Canada \\ Gordon Chan, University of Alberta, Edmonton, AB, Canada \\ Raja B. Singh, University of Alberta, Calgary, AB, Canada \\ Alan Underhill, University of Alberta, Edmonton, AB, Canada
}

